\title{
Pharmacists perceptions of community pharmacy practice in UAE: An Overview
}

\author{
Sarah Amir Al Akshar ${ }^{1}$, Dr. Mohammed Shamssain ${ }^{2}$ Prof. Zakia Metwaly ${ }^{3}$ \\ ${ }^{1}$ Lecturer, Ajman University of Science and Technology \\ ${ }^{2}$ Associate Professor, Ajman University of Science and Technology \\ ${ }^{3}$ Professor, Ajman University of Science and Technology
}

\begin{abstract}
There are limited published data available about the provision of pharmaceutical care in community pharmacies in the UAE. Almost no documentation of efforts was taking place in community pharmacy. Objectives: This study is done to evaluate pharmacists perception of their current practice, as well as their knowledge of drug regulations/ classification by the Ministry of Health (MOH). Also, investigate their opinion on the possible pharmacist patient communication barriers. Methods: 300 cross sectional surveys were distributed to community pharmacies in Ajman and Sharjah. In addition, a pharmacist played the role of a patient with a prescription to measure pharmacists' knowledge. Results: The response rate of the participants was 73.6\%. Most of the pharmacists couldn't identify drug- related problems in the prescription and dispensed antibiotic without a prescription. In addition, most pharmacists were satisfied by the dispensing role in the community pharmacy. Conclusion: Community pharmacists roles must be expanded and they must look for new challenges in their profession in order to improve their experience and capabilities.
\end{abstract}

KEYWORDS: community pharmacists, drug-related problems, obstacles, patient counseling, Pharmaceutical care.

\section{INTRODUCTION}

This study's main objective is the provision of the community pharmacy practice in UAE and exploring the extent of applying pharmaceutical care in the real life practice, in Ajman and Sharjah cities. Looking into the practice will help identify the defects, obstacles, barriers and misconceptions around pharmacists and their practice. Exploring the pharmaceutical care practice in community pharmacies and identifying the source of drug related problems will lead to prevention of problems and satisfy patients improving and strengthening the profession. This study will, also, explore antibiotics dispensing patterns as many studies done in UAE reported that dispensing antibiotics as an over the counter medications is a relatively frequent problem [1,2]. In addition, a limited published data is available about the provision of pharmaceutical care in community pharmacies in the UAE [3]. Almost no documentation of efforts was taking place in community pharmacy [4].

A study done in UAE by Aba Saeed et al. has found that among the 860 participants, 485 (56\%) have reported the use of antibiotics within the last year. Amoxicillin was the antibiotic most commonly used (46.3\%). Of the participants surveyed, 393 (46\%) stated that they intentionally use antibiotics as self-medication without a medical consultation, a behavior that is significantly affected by educational levels $(p<0.001)$. Two hundred forty-five (28\%) participants stored antibiotics at home. These antibiotics were mostly acquired from community pharmacies without prescriptions $(p<0.001)[1]$.

Many studies have been performed around the world to evaluate practice and the application of pharmaceutical care in community pharmacy setting $[5,6,7,8]$. A study done in Canada concluded that many pharmacists in community settings have not yet been able to implement pharmaceutical care as part of their routine work activities, a small number of pharmacists appeared to be introducing pharmaceutical care into the practice setting [5]. Another study performed by Toklu et al. evaluated the quality of pharmacy practice in 84 community pharmacies in the Umraniye district of Istanbul by face to face interviews and an unannounced simulated case scenario in 2010. The average dispensing time, dispensing practice, and adequate labeling were evaluated as rationality indicators. Results showed that $32 \%$ of the pharmacists were not present in their pharmacies during the simulation studies. Only $40.5 \%$ of the prescriptions were dispensed by the pharmacists. Forty four percent of the pharmacy employees had no more than a primary school degree. Half of the patients applying to a pharmacy had no prescription. The average dispensing time for a single drug was 149 seconds in simulated cases although the pharmacists declared 287 seconds in the questionnaires. All pharmacists reported that they explained to the patients how to administer their drugs but only $43 \%$ of the prescriptions were adequately labeled and only $6.5 \%$ of pharmacists included a verbal warning of possible interactions. In conclusion, good pharmacy practice was poorly applied in CP in the selected district of Istanbul [6]. 
In 2011, a study performed by Al-Hassan in Riyadh, Kingdom of Saudi Arabia (KSA), to determine the extent to which community pharmacists were engaged in patients counseling. A random sample of 100 pharmacies were selected and a questionnaire was designed and distributed to each pharmacy to obtain information to meet the study objectives. Only five of the total sample notified the possible drug interaction, even though most of the practitioners have stated that their education prepared them to monitor drug-drug interaction and they feel confident about this role. Furthermore, 95\% of pharmacists did not adhere to the profession legislation Act regarding antibiotic dispensing. Although a significant proportion of pharmacists have reported actual performance of such services and were willing to do so, the involvement of community pharmacists in providing patient counseling was minimal [7].

A study conducted by Parmar in Kenya and US, reported that when patients in Kenya walk into the pharmacy, they are usually just asked what medication/s they need and it's assumed that the physician had verified the allergies and looked at their medications for drug-drug interactions. The pharmacists usually do not verify this information. This is in contrast to the United States where a prescription is required in order to obtain a prescription medication, a physician's authorization would be needed to change a medication, and allergies and medication histories are taken from patients when they visit the pharmacy. In addition, the study stated that most pharmacists in Kenya indicated that even though prescription medications needed a prescription in order to be dispensed, the rules could be bent. Patients were able to get medications without prescription if they were able to afford them. This included controlled medications. For medications such as antibiotics, in Kenya, prescriptions were often not needed. In cases where patients were unable to afford the medications, pharmacists in Kenya were able to switch to a different medication without consulting a physician. Most pharmacists express that this was done because it would take too long for the physician to get back to them [9]. A study was done by Hawksworth et al. to examine the value of Clinical pharmacy provided by a community pharmacist during the dispensing process. It concluded that clinical pharmacy has the potential to provide a valuable contribution to health care. When normalized for the dispensing volume of each community pharmacy the lower the number of items dispensed then the greater was the percentage of interventions $(P=0.013)$. The clinical panel decided that between $19(0.01 \%$ of the total items dispensed) and $242(0.12 \%)$ interventions may have prevented a drug-related hospital admission, $71(0.04 \%)$ to $483(0.24 \%)$ could have prevented harm whilst $103(0.05 \%)$ to $364(0.18 \%)$ had the potential to improve the efficacy of the intended therapeutic plan. The panel also decided that $748(0.37 \%)$ interventions improved the clinical outcome and could have saved a visit to or by the general practitioner [10].

Another study done by Bryant et al. to investigate whether community pharmacists' perceptions of their role may be a barrier to increase their role in medication management. The results revealed a gap in perceptions regarding the role of the community pharmacist, with general acceptance of the technical roles but less acceptance of the clinical roles. It also observed that there was a lack of readiness to change by community pharmacists [11]. In United Kingdom (UK), a study done by Bond et al. to evaluate the system of managing repeated prescribing, it has been demonstrated to be logistically feasible, to identify clinical problems, and to make savings in the drugs bill. it was found in the study that A total of $12.4 \%$ of patients had compliance problems, side-effects, adverse drug reactions, or drug interactions identified by the pharmacist. There were significantly more problems identified in total in the intervention group. The total number of consultations, deaths, and non-elective hospital admissions were the same in both groups. Sixty-six per cent of the study patients did not require their full quota of prescribed drugs, representing $18 \%$ of the total prescribed costs (estimated annual drug cost avoidance of $£ 43$ per patient) [12]. A meta-analysis was done by Chisholm-Burns et al. to examine the effects of pharmacist-provided direct patient care on therapeutic, safety, and humanistic outcomes. It has concluded that Pharmacist-provided direct patient care had favorable effects across various patient outcomes, health care settings, and disease states. Incorporating pharmacists as health care team members in direct patient care is a viable solution to help improve US health care. In the study, favorable results were found in therapeutic and safety outcomes, and meta-analysis conducted for hemoglobin A1c, low density lipoprotein (LDL) cholesterol, blood pressure, and adverse drug events were significant $(P<0.05)$, favoring pharmacists' direct patient care over comparative services. Results for humanistic outcomes were favorable with variability. Medication adherence, patient knowledge, and quality of life-general health metaanalysis were significant $(P<0.05)$, favoring pharmacists' direct patient care [13].

A study done in Jordan by Al- Azzam et al. stated that out of the 9281 participants, 2133 (23\%) were using antibiotics over the study period: $842(39.5 \%)$ of them were self-medicating with antibiotics and 1291 had antibiotics prescribed for treatment. the main source of antibiotic supply were the previously prescribed antibiotics stored in the household (392) or purchased at retail pharmacies (370). Supplies by friends and relatives accounted for about 68 cases. The sources of information the participants needed for self-medication with antibiotics, such as drug selection and/or treatment regimen, were investigated. 
The survey results indicated that the participants relied mainly on their previous experience with the medication, and on pharmacist advice. To a lesser extent, they made use from the information available in leaflets, advice from relatives or friends, and physician consultation [14].

\section{OBJECTIVES}

Evaluate pharmacists perception of their current practice, as well as their knowledge of drug regulations/ classification by the Ministry of Health $(\mathrm{MOH})$. Also, investigate their opinion on the possible pharmacist patient communication barriers.

1. Evaluate pharmacists' pharmaceutical care practice in Ajman and Sharjah cities in the UAE by determining competence of pharmacists in monitoring drug-drug interactions, pharmacists' adherence to pharmacy regulations and the extent to which community pharmacists engage in patient counseling.

2. Evaluate pharmacists' pharmaceutical care practice in Ajman and Sharjah cities in the UAE by determining competence of pharmacists in monitoring drug-drug interactions, pharmacists' adherence to pharmacy regulations and the extent to which community pharmacists engage in patient counseling.

\section{METHODS}

To accomplish the study's objectives descriptive cross sectional surveys were used. The study was done during a period of eleven months, from August 2012 till June 2013. The source population were licensed community pharmacists working in Ajman and Sharjah to obtain the first and second study objectives. The inclusion criteria was; Registered licensed community pharmacists working at Ajman and Sharjah in 2012, Male and Female, Willing to participate in the study. While the Exclusion criteria excluded pharmacists Not willing to participate in the study, Pharmacists on leave during the study, Hospital pharmacists, Unlicensed community pharmacist or unregistered pharmacist in the $(\mathrm{MOH})$ and Ajman and Sharjah medical zone, Pharmacy technician and Trainee pharmacy students.

According to the $\mathrm{MOH}$ at the study period, there were a total of 1400 licensed community pharmacists in UAE in which around 500 licensed Community pharmacists working in Ajman and Sharjah in the year 2012. Multistage sampling method was chosen as it was the most economical data collection in the current study setting. In the first stage, the two cities of Ajman and Sharjah were selected by convenience sampling because of their convenient accessibility and proximity to the researcher. In the second stage, Simple random sampling applied for the selection of the community pharmacists in which all pharmacies in Ajman and Sharjah mentioned in the MOH list were given numbers. All pharmacies' numbers were placed in a box and shaken to ensure randomization. Numbers were picked until the completion of 300 pharmacies. One pharmacist was selected from each pharmacy during the visit by inviting the available licensed community pharmacist to participate. The same pharmacist who was invited to fill the survey was asked to dispense the prescription. The sample size was calculated on steps by referring to similar studies conducted in Kenya and Saudi Arabia [7, 9], to ensure achievement of maximum response and reliable statistical sample size. The minimum sample size was calculated using "Raosoft" online sample size calculator (218n) (Available at: http://www.raosoft.com/samplesize.html. The population of licensed community pharmacists in Ajman and Sharjah UAE (500) at 2012, confidence level of 95\% and 5\% margin of error were used in the calculation. Fifteen percent added to the minimum sample size to avoid decreased sample size than the minimum $(n=250)$. The final chosen sample size was 300 to overcome non response. Seven recruited pharmacists were requested to distribute and collect the samples with the researcher. The 7 pharmacists were trained to administer the questionnaire in a face-to face interview; they were trained for 2 hours in an accumulative session in order to adopt understanding and uniform questionnaire administration. Two of the seven recruited pharmacists visited each pharmacy between October 2012 and January 2013, and invited the licensed pharmacist for participation.

The questionnaire was designed by the researcher based on the parameters to be evaluated as a part of the study and by referring to previous literature [7]. The initial drafts were made and circulated to the supervisor and modifications were carried out as per the suggestions ensuring convenience for community pharmacists in UAE. Face validity was done by university lecturers and experts. The questionnaire was designed to be an interview administrated and to be completed in 10 minutes and covered four parts; Socio-demographic characteristics, evaluating pharmaceutical practice by pharmacists, pharmacists knowledge on federal regulations, and exploring pharmacists perception on pharmacists patient communication. Items were worded as a series of statements and the pharmacists were asked to indicate their agreement or disagreement on a 4-point likert scale from 'strongly agree' to 'strongly disagree'. Items were collapsed into three categories; agree, neutral, and disagree. A final open-ended question invited the respondents to suggest possible ways to improve community pharmacist and patient relationship. 
The questionnaire was initially developed in English language and translated into Arabic by two native Arabic pharmacists. Another two native Arabic pharmacists translated the questionnaire back to English to ensure accuracy. For investigating pharmacists' pharmaceutical care practice and adherence to regulations, a prescription was designed by the researcher based on the parameters to be evaluated as a part of the study and by referring to previous literature [7]. The prescription was designed to measure pharmacists' ability to identify drug- drug interactions, doses of medications, antibiotic dispensing patterns and whether pharmacists follow $\mathrm{MOH}$ regulations. As well as, evaluate pharmacists activity in counseling patients who have chronic diseases including side effects and other possible drug related problems, educating patients on medications use instructions. Also to measure pharmacist communication with the patient as well as pharmacist physician relations. As well as pharmacists' knowledge of the appropriate dosing.

The prescription contained the following medications; Aspirin 81, Perindopril $4 \mathrm{mg}$, Janumet 50/1000 $\mathrm{mg}$, Pravastatin 20mg, Neurobion, Bisoprolol 5, Celecoxib 100mg. Also, the observer, a pharmacist playing the role of a patient asked for Augmentin 1g. If the observer was asked about the age of patient, the answer would be nine years old. A form was made to help the observer evaluate practice and adherence of community pharmacists .

This prescription was intended to cover 5 main parts; whether the pharmacist provided any information for the patient about diabetes mellitus or hypertension, check if the pharmacist provided any instructions or information about the medications dispensed, whether the pharmacist suspected that the prescription was fake, whether the pharmacist noticed the drug-drug interactions in the prescription or not. Also, it recorded the reaction of the pharmacist who counselled the observer about possible drug related problem and whether the pharmacist called the physician and discussed substitution, whether the pharmacist consulted the physician or not, and if the pharmacist substituted the medication or dispensed it anyway without giving any proper solution. The last part covered $\mathrm{MOH}$ regulations regarding antibiotic dispensing and whether the pharmacist dispensed the antibiotic without the prescription or not. In case the pharmacist decided to dispense the medication, the observer recorded whether the pharmacist asked any questions before the antibiotic was dispensed or not, especially, the age and weight of the patient. If the pharmacist asked, the patient would respond it is for a 9 year old child, then the pharmacist reaction was recorded to whether the antibiotic dose was adjusted to patient's details or not.

Also, a pilot study was conducted to ten pharmacists/ patients to test the validity and the reliability of the survey form, to determine the time needed for data collection and to revise and finalize the questionnaire. Feedback given by the pharmacists/ patients were considered and corrections were made accordingly. Questions adjustments were made to both questionnaires to improve validity as well as answering the researcher's questions of the study. Originally the questionnaires were designed to be self-administrated but based on the lower response during the pilot study, decision was made by the researcher to modify the questionnaires from self -administrated questionnaires to face to face questionnaires.

Also, Pharmacists willing to participate in the study were provided with the study information sheet with a clear fully verbal explanation about the study and in addition they were requested to sign the informed consent form. Individual Face to face questionnaire, interview questionnaire, was used. Participation was voluntary and no incentives were given to the participants. The interviewer intervened only to clarify a question, if required. No attempt was made to prompt the respondents by suggesting answers directly. In addition, a pharmacist playing the role of the patient with the prescription didn't ask any questions and only handed the community pharmacist the prescription.

In this study the data analysis was conducted using SPSS version 20. Instituting identification numbers were given for all questionnaires. All questions were coded and then they were imported for SPSS for analysis. All variables categories were coded with numbers. The items were checked for accuracy by examining unusual coding values and $10 \%$ of returned surveys were randomly selected for hand checking by an independent person. Descriptive analysis was used including mean, median, standard deviation and frequency. Results were presented as numbers with percentages or graphic presentations for categorical variables.

Ethical standards for conducting the study were maintained as follows, Permission to conduct the study was requested and obtained from Ajman and Sharjah Ministry of Health, Informed consent from participants was obtained prior to conducting the study, Confidentiality of participants was maintained at all times, Participants' information obtained from the questionnaires was kept confidential, Participants' were informed that participation is voluntary and they could withdraw from the study at any stage. 


\section{IV- RESULTS}

The response rate was $73.6 \%$ in which out of the 300 questionnaires distributed the total number of usable data was 221 . Fifty nine pharmacists (19.6\%) were not willing to participate in the study. The remaining 221 were analyzed.

Table 4.1: Socio-demographic data of community pharmacists.

\begin{tabular}{|c|c|c|c|}
\hline \multirow[t]{2}{*}{ Characteristics } & \multicolumn{3}{|c|}{$\mathrm{N}(\%)$} \\
\hline & Female & Male & Total \\
\hline Gender & $97(43.9)$ & $124(56.1)$ & $221(100)$ \\
\hline \multicolumn{4}{|l|}{ Age } \\
\hline$<30$ years & $47(48.5)$ & $32(25.8)$ & $79(35.7)$ \\
\hline $30-50$ years & $47(48.5)$ & $78(62.9)$ & $125(56.6)$ \\
\hline$>\mathbf{5 0}$ years & $3(3.1)$ & $14(11.3)$ & $17(7.7)$ \\
\hline \multicolumn{4}{|l|}{ Nationality } \\
\hline UAE & $0(0)$ & $0(0)$ & $(0)$ \\
\hline Southeastern Asia & $25(25.8)$ & $74(59.7)$ & $99(44.8)$ \\
\hline Arab & $59(60.8)$ & $25(20.2)$ & $84(38.0)$ \\
\hline Others & $13(12.2)$ & $25(20.2)$ & $38(16.3)$ \\
\hline \multicolumn{4}{|l|}{ Years of experience } \\
\hline$<5$ years & $52(41.2)$ & $47(37.9)$ & $99(37.1)$ \\
\hline 5-10 years & $30(30.9)$ & $39(31.5)$ & $69(31.2)$ \\
\hline$>10$ years & $15(15.5)$ & $38(30.6)$ & $53(24)$ \\
\hline \multicolumn{4}{|l|}{ Sitting of pharmacy } \\
\hline Chain pharmacies & $46(47.4)$ & $59(47.6)$ & $105(47.5)$ \\
\hline Independent pharmacies & $51(52.6)$ & $65(52.4)$ & $116(52.5)$ \\
\hline
\end{tabular}

From table 4.1, pharmacists were mainly males, 124 (56.1\%), and most of the pharmacists, 125 $(56.6 \%)$, were between age 30 to 50 years.

The majority of the community pharmacists, 213(96.4\%), participating in the study stated that patients come back frequently to their pharmacies which makes patients' follow up possible. Most pharmacists participating, 149(67.4\%), were available in their pharmacies for 7 - 9 hours per day. Most pharmacists, $152(68.8 \%)$, claimed that they spend 1-4 hours per day counseling patients, also, $196(88.7 \%)$, stated that they spend less than 10 minutes with each patient. Majority of pharmacists, $176(79.6 \%)$, reported that they have no comprehensive source of patients' medical history and around, $159(71.9 \%)$, have no records that can be used for tracking patients to have contact with them for follow up.

Table 4.2: Pharmacists' evaluation criteria when prescriptions were distributed.

\begin{tabular}{|ll|}
\hline Pharmacists were monitored for & $\mathbf{N}(\%)$ \\
\hline Giving instructions on how to use medications & $3(1.4 \%)$ \\
\hline Educating patients about disease & $3(1.4 \%)$ \\
\hline Identifying drug-drug interactions & 0 \\
\hline Educating patients on medications' side effects & 0 \\
\hline Dispensing antibiotic without a prescription & $206(96.3 \%)$ \\
\hline Asking about the patient's age and other details & $24(11.2 \%)$ \\
\hline Changing the dose according to patient's details & $16(7.5 \%)$ \\
\hline
\end{tabular}

From table 4.2 , out of the 218 pharmacists, who received a prescription by a pharmacist who played the role of a patient, 206(96.3\%), pharmacists dispensed antibiotic when asked for it verbally. None of the pharmacists educated observer about the potential drug interaction nor side effects of the prescription medications. 


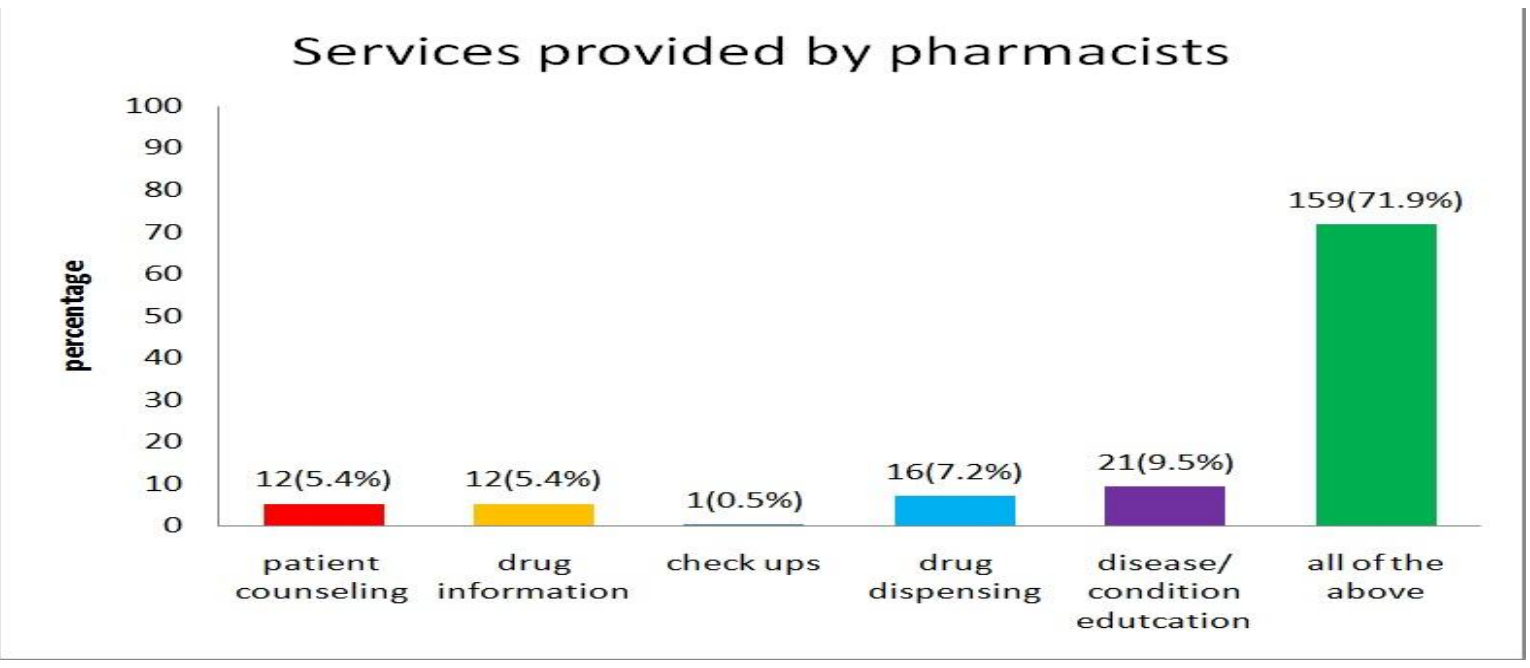

Fig. 4.1: services offered by community pharmacists

From figure 4.1, when pharmacists were asked about the services they provide to patients, 159 (71.9\%), of pharmacists claimed that they provide all the mentioned pharmaceutical care services; patient counseling, drug interactions, checkups, drug dispensing instruction, disease or condition education services. While only, 1 $(0.5 \%)$ of community pharmacists provide checkups.

Table 4.3: pharmacists perception of pharmaceutical care services.

\section{Statement}

$\mathbf{N}(\%)$

I am satisfied with the amount of time spent with each

$\begin{array}{lll}\begin{array}{l}\text { Strongly agree and } \\ \text { agree }\end{array} & \text { Neutral } & \begin{array}{l}\text { Disagree and } \\ \text { strongly disagree }\end{array} \\ 201(91) & 13(6) & 7(3)\end{array}$

patient when they come in

\begin{tabular}{|c|c|c|c|}
\hline $\begin{array}{l}\text { I am satisfied with the Information provided to patients } \\
\text { on how to use the drug }\end{array}$ & 202(91.4) & $7(3)$ & $12(5.6)$ \\
\hline $\begin{array}{l}\text { I am satisfied with the level of drug interaction } \\
\text { counseling available to patients }\end{array}$ & $168(76)$ & $14(6)$ & $39(17)$ \\
\hline $\begin{array}{l}\text { I am satisfied with the overall operations of } \\
\text { pharmacies/chemists }\end{array}$ & $175(79.2)$ & $16(7.2)$ & $48(21.6)$ \\
\hline $\begin{array}{l}\text { I am satisfied with the education/training provided to } \\
\text { chemists/pharmacists }\end{array}$ & $185(83.7)$ & $9(4)$ & $27(12.3)$ \\
\hline $\begin{array}{l}\text { I am satisfied with the information about allergy given } \\
\text { to patients before dispensing a medication. }\end{array}$ & $193(87.3)$ & $4(1.8)$ & 24(10.9) \\
\hline $\begin{array}{l}\text { I am satisfied with the history of medication taken from } \\
\text { patients before dispensing a medication. }\end{array}$ & $180(81.4)$ & $13(6)$ & $28(12.6)$ \\
\hline $\begin{array}{l}\text { I am satisfied with the average understanding a patient } \\
\text { has about a medication }\end{array}$ & $181(82)$ & $7(3)$ & $33(15)$ \\
\hline I am satisfied with the quality of medications available. & $194(87.8)$ & $12(5.4)$ & $15(6.7)$ \\
\hline I am satisfied with the level of medication regulation & $190(86)$ & $6(2.7)$ & $25(11.3)$ \\
\hline I am satisfied with the cost of medications & $36(16)$ & $31(14)$ & $154(70)$ \\
\hline
\end{tabular}

I am satisfied with the cost of medications

From table 4.3, The majority of pharmacists were satisfied with the information they provide to patients; 202(91\%). Seventy six percent (168) of the pharmacist were satisfied with the level of drug interaction counseling available to patients. Seventy percent (154) of pharmacists are not satisfied with the cost of medications 


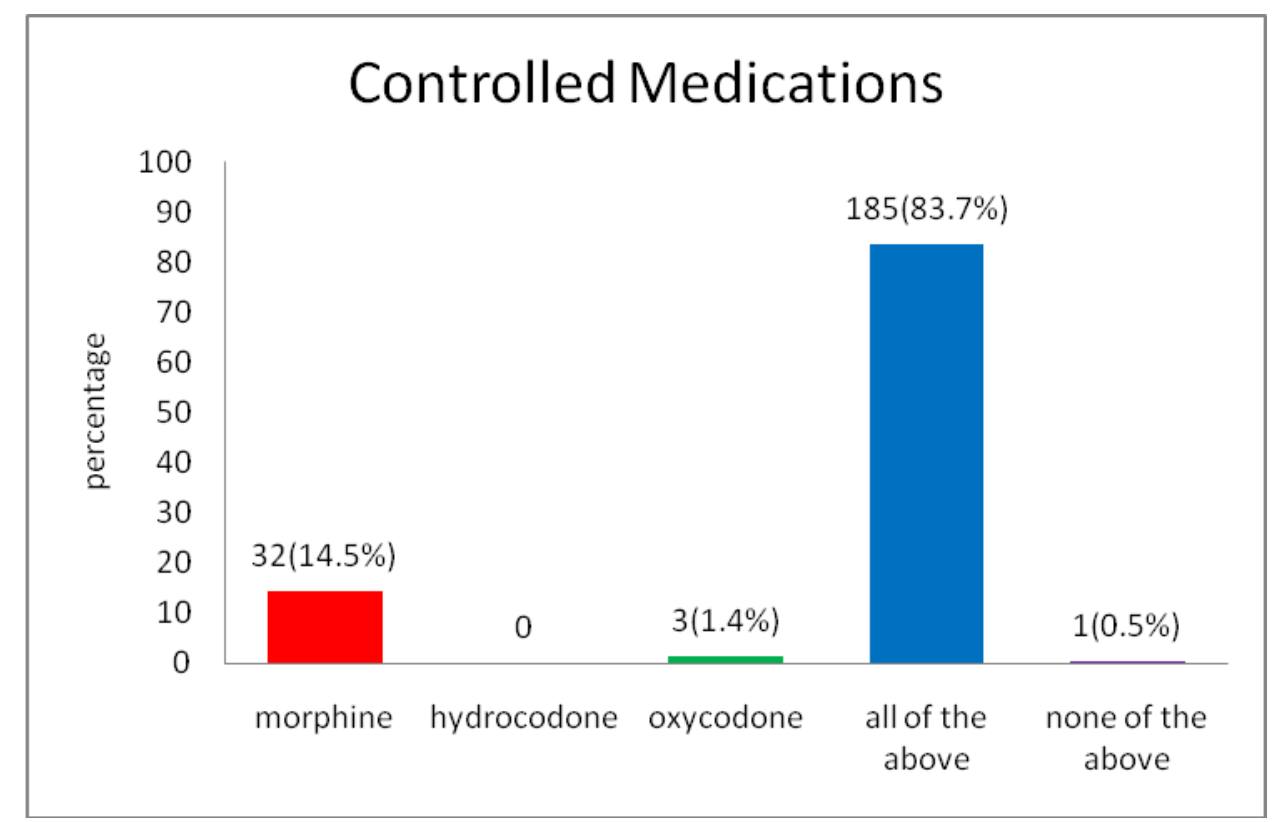

Fig. 4.2: controlled medications according to community pharmacists knowledge

From figure 4.2, 185 (83.7\%), of pharmacists, thought that morphine, hydrocodone and oxycodone are controlled medications. While only, $1(0.5 \%)$ of pharmacists thought that none of the medications were controlled.

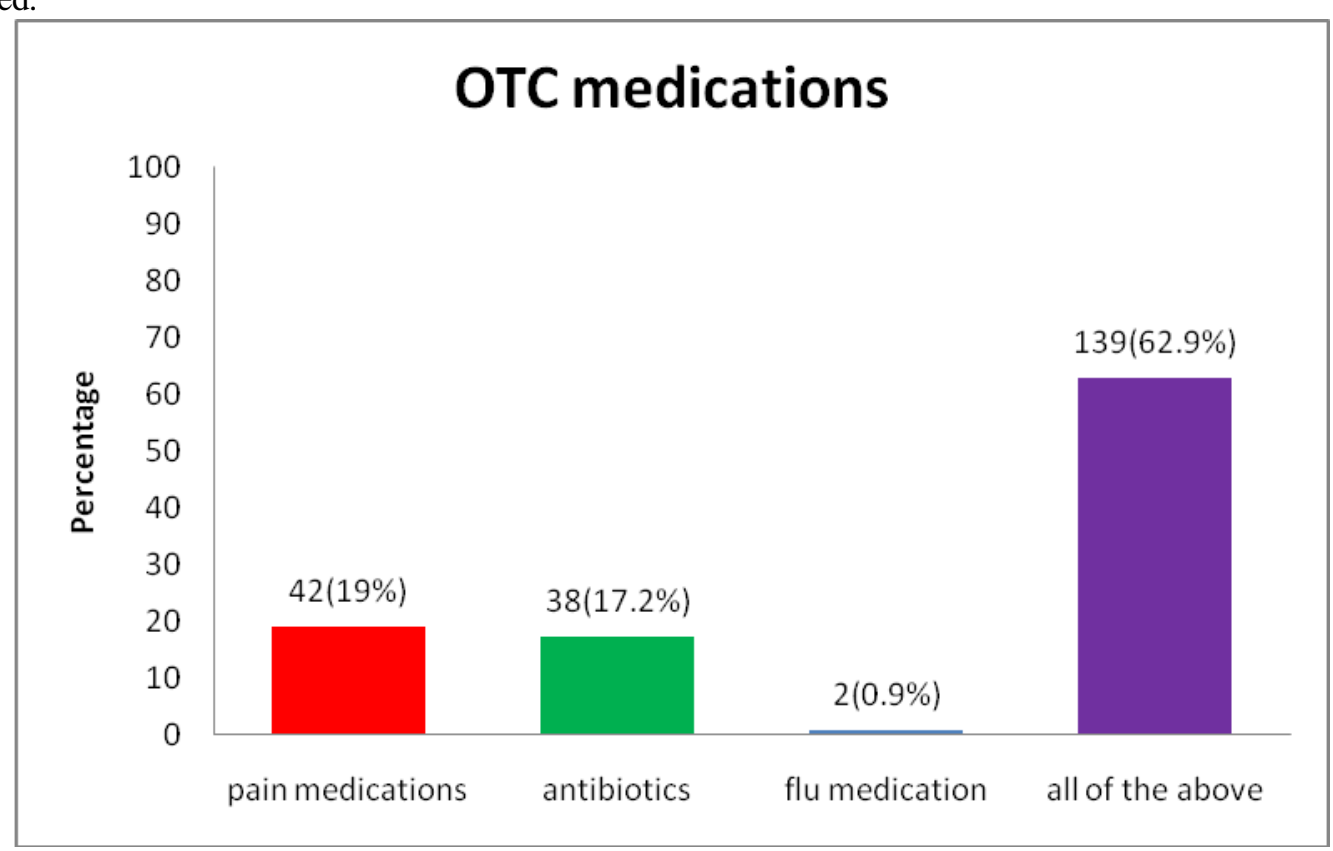

Fig. 4.3: OTC medications according to community pharmacists knowledge

From figure 4.3, around sixty three percent, 139, thought that pain, antibiotics and flu medications are OTC medication 


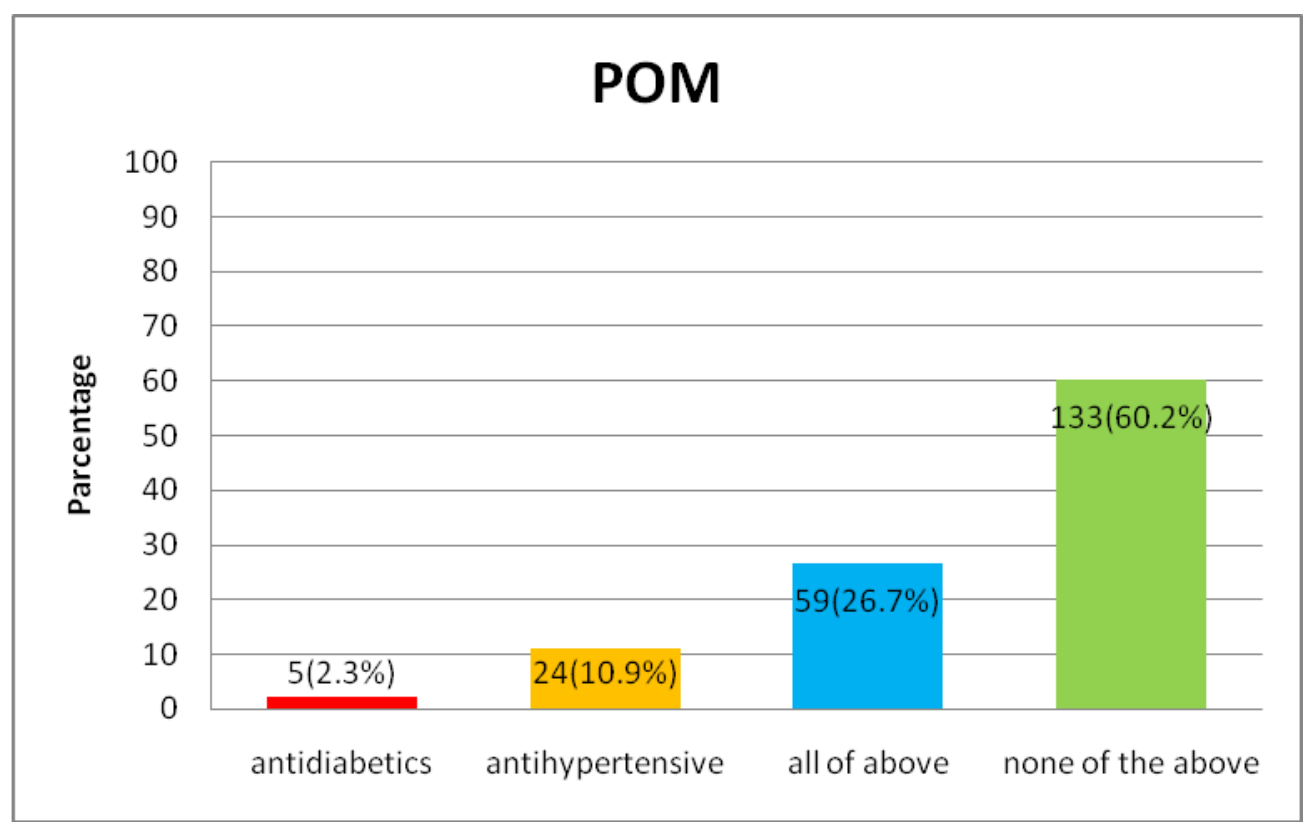

Fig. 4.4: POMs according to community pharmacists knowledge

From figure 4.4, 109 (49.3\%), of pharmacists believed that anti-diabetics and anti-hypertensive medications don't require a prescription.

\section{DISCUSSION}

This study is one of the very few studies done in the UAE to investigate the practice of community pharmacists, with respect to pharmaceutical care services. Also, to evaluate community pharmacists' knowledge regarding medications and regulations in UAE, as well as, the perceptions of community pharmacists towards providing pharmaceutical care services. Finally, suggest some of the reasons of the almost diminished involvement of community pharmacists in providing patient care. The majority of pharmacists were satisfied with the information they provided to patients, 202 (91\%). Seventy six percent of the pharmacist (168), were satisfied with the level of drug interaction counseling available to patients. This is similar to the results of other studies done by Parmat in Kenya and USA and Bryant et al. in New Zealand [9, 11]. These results oppose to the results of a study by El Hajj in Qatar which found that only 31\%, 29\%, 17\%, of community pharmacists believed that they provide enough medications counseling, enough use instructions and appropriate monitoring, respectively [8]. Community pharmacists can play a critical role in improving the quality of facilities they render to fulfill the requirements and the desires of patients by providing high standard pharmaceutical care. However, it is the responsibility of policy makers to keep up the positive perception of pharmacists and to encourage the practice of pharmaceutical care activities.

In addition, hundred and fifty nine pharmacists (72\%) participating in this study claimed that they provide all of the following pharmaceutical care services; patient counseling, drug interactions, checkups, drug dispensing instruction, disease or condition education services. But only, 3 (1.4\%), pharmacists provided patient counseling and drug dispensing instructions after receiving the prescription. This is compatible with another study by Al-Hassan which reported that $82 \%$ of pharmacists felt that their education prepared them to monitor drug-drug interactions, $78 \%$ felt confident for this role and $75 \%$ of the surveyed pharmacists reported a routine scanning for possible drug interaction prior to dispensing any requests [7]. It is a serious concern to recognize that large percentage of the pharmacists didn't practice patients counseling which may impact negatively on the public health and contribute to increase medication failure. These findings advocate the need for awareness program for the pharmacists about counseling practice importance and the impact of patients' health life. A possible explanation of the lack of pharmacists' ability to identify drug related problems could be due to limited immediate access to up-to-date resources [4].

In this study it was clear that pharmacists perceive their current practice and involvement in pharmaceutical care much higher than what they really provide to patients. These results confirm the results of other studies in New Zealand and Turkey $[6,15]$. Many pharmacists do not regularly counsel patients and when they do, the extent and quality of counseling by pharmacists is inadequate [7]. A hundred and ninety six pharmacists $(89 \%)$ reported that they spend less than 10 minutes with each patient. This is much less than the range claimed by pharmacists in Riyadh, KSA, who claimed average of 28 minutes of counseling per patient 
[7]. Definitely proper patient counseling practice can be implemented through establishment of continuous educational workshops and conferences for community pharmacists. Almost 176 of pharmacists, (79.6\%), reported that they have no comprehensive source of patients' medical history and, $159(71.9 \%)$, stated that they have no records that can be used for tracking patients to have contact with them for follow up. These results are incompatible with the results of Parmar who found that pharmacists in Kenya and USA keep a paper based records, automated records of patients [9]. These results urgently emphasize the need for the decision makers in UAE to develop strategies in order to facilitate pharmacists' follow up process to patients.

A hundred and sixty eight $(76 \%)$ of pharmacists participating in this study claimed that they intervene when finding a drug related problem in a prescription. But when given a prescription with drug related problems, none of the pharmacists intervened nor counseled the patient about drug-drug interaction nor side effects of medications. This is similar to the results of a study by Al- Hassan, where only five pharmacists identified and intervened the drug interaction in the request made by the patient [7]. This is also similar to findings of another study done by Carter et al. which found out that only $0.7 \%$ of pharmacist in Iowa, US intervene when finding a drug related problem in a prescription [16]. It is mandatory for the pharmacist educational program to focus on DRP management.

Also, none of the pharmacists, participating in this study, who dispensed the request indicated any dosage directions or when medications should be taken with regard to meals. In addition, none of the pharmacists told the observer to continue taking the antibiotic until all the course is over, and none of the pharmacists warned the observer about potential problems caused by taking certain foods (e.g., grape) herbs (e.g., St. Johns') or other drugs (e.g., celebrex) in conjunction with the medications in the prescription $[17,18]$. These results are compatible with the results of Al- Hassan where only 5\% educated the observer about potential DRPs [7]. These results should advocate pharmacy council to include DRP management and patient counseling in continuous pharmaceutical education as a part of license requirements for pharmacists in UAE.

In this study, only 24(11\%), of the pharmacists asked the observer who the prescription was for, the age and other patient details before dispensing the medications to the observer. Unlike results of a study [19] in Denmark which reported only $2 \%$ of pharmacists don't know patient details before dispensing. This result showed the wide spread of not knowing the patient before dispensing the medications as it increases the chance of having a drug related problem such as giving inappropriate dose the patient. This calls UAE policy makers for an immediate intervention by implementing stronger rules regarding dispensing of POM specifically and drugs generally.

In this study, when evaluating the knowledge of pharmacists on POM and OTC medications, unfortunately, 139 (62.9\%), of the pharmacists participating in this study thought that antibiotics, analgesics, flu medications are OTC medications. Also, 109(49.3\%) of pharmacists participating in this study believed that anti-diabetics, anti-hypertensives are not POMs. This is similar to a study carried out in Saudi Arabia, where 85.4\% of pharmacists are confused between POM and OTC medications [20]. In spite of the fact that antibiotics are under POM category, this study found that the observer, who played the role of a patient, received antibiotics after a verbal request without a prescription form, 206 (96.3\%), pharmacies out of the 218 pharmacies participating in this study. This rate is very close to the rate of dispensing antibiotic without a prescription in KSA, which reached $95 \%$ in a study by Al- Hassan [7]. This is higher than the rate published in other studies in Eastern Europe, UAE and Jordan [1, 14, 21] which found that the rate is 68\%, 56.3\% and 39.5, respectively. These results raise the need of urgent intervention to improve community pharmacists' knowledge about the regulations and drug classifications. An educational program can include presentations, workshops and small group discussions, providing information by mail, newsletters, reminders, advertisement and continuous education of pharmacists.

Patient- pharmacist communication is reported to be a cause of improving patient medication use, health outcomes and satisfaction [22]. In an open end question about the suggestions of improving patientpharmacist communication, some of the pharmacists suggested solutions to overcome the barriers. From pharmacists perspective, the barriers could be solved by more training, experience and education, less legal restrictions on the services to be provided such as monitoring blood pressure and blood glucose, having patient records and information, and improved attitude towards the role of pharmacists by patients and physician. These results are similar to the results of other studies examining barriers [7,23,24]. The challenge thus remains to policy makers and regulatory authority to put more efforts to provide pharmaceutical care services, by providing appropriate and suitable training and opportunities for community pharmacists as they have tendency to attend training programs. 


\section{CONCLUSION}

Pharmacists should know that providing pharmaceutical care services to patients during the dispensing process has the potential to provide a valuable contribution to health care. Improving perceptions and attitudes of patients, pharmacists and physicians towards the role of pharmaceutical care in improving patient overall health is essential. Community pharmacists roles must be expanded and they must look for new challenges in their profession in order to improve their experience and capabilities.

\section{LIMITATIONS}

A convenience sample was used for this study and may not fully represent the whole public in UAE. Our focus was on describing perceptions held by people who use community pharmacies only. Further studies on other populations like those who use hospital pharmacies is warranted. As this was a voluntary survey, the responses may have contained self-reporting data inaccuracies resulting from intentional deception, poor memory, or misunderstanding of the question. Therefore, findings of this study should be interpreted with the limitations in mind.

\section{REFERENCES}

[1]. Abasaeed, A., Vlcek, J., Abuelkhair, M., \& Kubena, A. (2009). Self-medication with antibiotics by the community of Abu Dhabi Emirate, United Arab Emirates.The Journal of Infection in Developing Countries, 3(07), 491-497.

[2]. Dameh, Majd, James Green, and Pauline Norris. "Over-the-counter sales of antibiotics from community pharmacies in Abu Dhabi." Pharmacy world \& science 32.5 (2010): 643-650.

[3]. Fahmy, S. A., Abdu, S., \& ABUELKHAIR, M. (2010). Pharmacists' attitude, perceptions and knowledge towards the use of herbal products in Abu Dhabi, United Arab Emirates. Pharmacy Practice, 8(2), 109-115.

[4]. Hasan, S., Sulieman, H., Chapman, C., Stewart, K., \& Kong, D. (2011). Community pharmacy in the United Arab Emirates: characteristics and workforce issues. International Journal of Pharmacy Practice, 19(6), 392-399.

[5]. Ramaswamy-Krishnarajan, J., \& Hill, D. S. (2005). Pharmaceutical Care in Canada An Exploratory Study of 81 Community Pharmacies. Canadian Pharmacists Journal/Revue des Pharmaciens du Canada, 138(4), 46-50.

[6]. Toklu, H. Z., Akici, A., Oktay, S., Cali, S., Sezen, S. F., \& Keyer-Uysal, M. (2010). The pharmacy practice of community pharmacists in Turkey. Marmara Pharm J, 14, 53-60.

[7]. Al-Hassan, M. I. (2011). Community Pharmacy Practice In Saudi Arabia: An Overview. The Internet Journal of Pharmacology, 9(1).

[8]. El Hajj, M. S., Salem, S., \& Mansoor, H. (2011). Public's attitudes towards community pharmacy in Qatar: a pilot study. Patient preference and adherence,5, 405-422.

[9]. Parmar, S. (2008). Community Pharmacy Practice in the United States and Kenya: Comparison. Diabetes in Control. com, 408, $1-9$.

[10]. Hawksworth, G. M., Corlett, A. J., Wright, D. J., \& Chrystyn, H. (1999). Clinical pharmacy interventions by community, pharmacists during the dispensing process. British journal of clinical pharmacology, 47, 695-700.

[11]. Bryant, L. J., Coster, G., Gamble, G. D., \& McCormick, R. N. (2009). General practitioners' and pharmacists' perceptions of the role of community pharmacists in delivering clinical services. Research in Social and Administrative Pharmacy, 5(4), 347-362.

[12]. Bond, C., Matheson, C., Williams, S., Williams, P., \& Donnan, P. (2000). Repeat prescribing: a role for community pharmacists in controlling and monitoring repeat prescriptions. The British Journal of General Practice,50(453), 271-275.

[13]. Chisholm-Burns, M., Lee, J. K., Spivey, C., Slack, M., Herrier, R., Hall-Lipsy, E., \& Wunz, T. (2010). US Pharmacists' Effects as Team Members on Patient Care: Systematic Review and Meta-Analyses. Medical care, 48, 923-933.

[14]. Al-Azzam, S. I., Al-Husein, B. A., Alzoubi, F., Masadeh, M. M., \& Al-Horani, M. A. S. (2007). Self-medication with antibiotics in Jordanian population.International journal of occupational medicine and environmental health, 20(4), 373-380.

[15]. Iversen, L., Mollison, J., \& MacLeod, T. N. N. (2001). Attitudes of the general public to the expanding role of community pharmacists: a pilot study. Family practice, 18(5), 534-536.

[16]. Carter, B. L., Chrischilles, E. A., Scholz, D., Hayase, N., \& Bell, N. (2003). Extent of services provided by pharmacists in the Iowa Medicaid Pharmaceutical Case Management program. Journal of the American Pharmacists Association, 43(1), $24-33$.

[17]. Llorca, C. S., Serra, M. P. M., \& Donat, F. J. S. (2008). Interactions between ibuprofen and antihypertensive drugs: Incidence and clinical relevance in dental practice. studies, 13(11):E717-21.

[18]. Alkharfy, K. M. (2010). Community pharmacists' knowledge, attitudes and practices towards herbal remedies in Riyadh, Saudi Arabia. EMHJ, 16(9), 988-993.

[19]. Knudsen, P., Herborg, H., Mortensen, A. R., Knudsen, M., \& Hellebek, A. (2007). Preventing medication errors in community pharmacy: frequency and seriousness of medication errors. Quality and Safety in Health Care, 16(4), 291-296.

[20]. Bawazir, S. A. (1992). Prescribing pattern at community pharmacies in Saudi Arabia. International Pharmacy Journal: 6 (5);222-224.

[21]. Grigoryan, L., Haaijer-Ruskamp, F. M., Burgerhof, J. G., Mechtler, R., Deschepper, R., Tambic-Andrasevic, A., \& Birkin, J. (2006). Self-medication with antimicrobial drugs in Europe. Emerging infectious diseases, 12(3), 452-459.

[22]. Schommer, J. C., \& Wiederholt, J. B. (1995). A field investigation of participant and environment effects on pharmacist-patient communication in community pharmacies. Medical Care, 567-584.

[23]. McKenzie, P. J. (2002). Communication barriers and information-seeking counterstrategies in accounts of practitioner-patient encounters. Library \& Information Science Research, 24(1), 31-47.

[24]. Hughes, C. M., \& McCann, S. (2003). Perceived interprofessional barriers between community pharmacists and general practitioners: a qualitative assessment. The British journal of general practice, 53(493), 600-606. 